\title{
Combination of epidural spinal cord stimulation and treadmill training with body weight support attenuates spasticity in adult rats with spinal cord transection
}

\section{Junya Saito ( $\boldsymbol{\nabla}$ j_sai_to@yahoo.co.jp )}

Toho University Medical Center Sakura Hospital https://orcid.org/0000-0001-9170-9135

\section{Masao Koda}

Department of Orthopaedic Surgery, Faculty of Medicine, University of Tsukuba

\section{Takeo Furuya}

Department of orthopaedic surgery, Chiba University Graduate School of Medicine

\section{Yasushi lijima}

Department of Orthopaedic Surgery, Chiba University Graduate School of Medicine

\section{Mitsuhiro Kitamura}

Department of Orthopaedic Surgery, Chiba University Graduate School of Medicine

\section{Takuya Miyamoto}

Department of Orthopaedic Surgery, Chiba University Graduate School of Medicine

\section{Chihiro Kai}

Department of rehabilitation, Chiba Rehabilitation Center

\section{Sumihisa Orita}

Department of Orthopaedic Surgery, Chiba University Graduate School of Medicine

\section{Kazuhide Inage}

Department of Orthopaedic Surgery, Chiba University Graduate School of Medicine

\section{Masashi Yamazaki}

Department of Orthopaedic Surgery, Faculty of Medicine, University of Tsukuba

\section{Seiji Ohtori}

Department of Orthopaedic Surgery, Chiba University Graduate School of Medicine

\section{Research article}

Keywords: spinal cord injury, spinal cord stimulation, treadmill training, spasticity, GAD65

Posted Date: May 11th, 2020

DOI: https://doi.org/10.21203/rs.3.rs-21128/v1 
License: (c) (i) This work is licensed under a Creative Commons Attribution 4.0 International License. Read Full License 


\section{Abstract \\ Background}

The neural control of rhythmical hindlimb locomotion is known to be organized by a central pattern generator (CPG) in the lumbar enlargement. In the previous report, epidural spinal cord stimulation (SCS) for CPG with treadmill training facilitated restoration of locomotion in patient with motor incomplete spinal cord injury. However, the precise mechanism of improvement is still unclear. The purpose of this study was to elucidate efficacy and molecular mechanism of SCS with treadmill training.

\section{Methods}

Ten female Sprague-Dawley rats were used in this study. The rats were housed with food and water under a $12 \mathrm{~h} / 12 \mathrm{~h}$ light-dark cycle. We performed spinal cord transection at the T8-T9 level. After two weeks, the rats were divided into SCS group and control group. The rats in SCS group were performed implantation of an electrode at L2 level. SCS and body weight support treadmill training were performed for 30 minutes per day, 5 days per weeks, for 4 weeks. The rats in the control group were performed treadmill training without stimulation for same period. We evaluated hind-limb locomotor function by using BassoBeattie-Bresnahan (BBB) score. We also assessed resistance force for full extension of hindlimb as evaluation of spasticity. For histological examination, we performed immunostaining for glutamic acid decarboxylase-65 (GAD65) of lumbar enlargement after behavioral assessment.

\section{Results}

There was no significant difference of BBB score between two groups, but spasticity of hind-limb in SCS group was significantly reduced. Histological assessment revealed that expression of GAD65 tended to increase in the SCS group

\section{Conclusions}

SCS ameliorated hindlimb spasticity. It was suggested that up-regulation of GAD65 involved suppression of spasticity.

\section{Background}

The neural control of rhythmical hind limb locomotion is organized by a neural circuit in the lumbar enlargement called the central pattern generator (CPG) [1]. The CPG consists of a separate network for rhythm generation and a network for pattern formation that deliver reciprocal excitation and inhibition to motoneurons. The activity of the CPG is autonomous because limb movement can be generated even when the spinal cord is totally transected and the dorsal roots are cut [2], and is modulated by descending 
supraspinal pathways and ascending afferents from the periphery under normal conditions [3]. Inability of hind limb locomotion after spinal cord injury (SCI) is attributed to CPG modulation failure because of a lack of supraspinal input. Therefore, CPG activation is a promising strategy to restore locomotor function after SCl.

Epidural spinal cord stimulation (SCS) for CPG-evoked bilateral hind limb locomotor activity in rats and contact of the hind limb with a treadmill belt is a necessary condition for locomotor activity [4]. Step training and the 5-HT agonist quipazine are known to facilitate stepping with epidural stimulation $[5,6]$. In humans, Herman et al. reported that combined epidural SCS and partial weight-bearing therapy could facilitate restoration of functional locomotion in patients with a $C$ rating on the American Spinal Injury Association Impairment Scale (AIS) [7]. In patients with a B rating on the AIS, epidural SCS for CPG enabled full weight-bearing standing and stepping [8]. To date, the precise mechanism of functional improvement induced by SCS and treadmill training remains unclear.

The purpose of the present study was to elucidate the efficacy and molecular mechanism of SCS for CPG using treadmill training with body weight support.

\section{Methods}

\section{Animals}

All experimental procedures were approved by the Animal Ethics Committee of Chiba University Hospital (approval No. A28-090, 29 - 10). Ten female Sprague Dawley rats (8-9 weeks old) were used in the present study. A summary of the experimental protocol is shown in Fig. 1.

\section{Surgical Procedure}

All rats were anesthetized with $2 \%$ sevoflurane (Sevofrane, Maruishi Pharmaceutical Co., Osaka, Japan) delivered via a nose cone and deep level of anesthesia was maintained during surgical procedures. We performed laminectomy at the level of T8-9 and completely transected the spinal cord. To prevent reconnection of the cut ends of the spinal cord, we resected a segment of the spinal cord at the T8-9 level. We used a small cotton pellet and microfibrillar collagen hemostat (Avitene, C. R. Bard, Murray Hill, NJ) for hemostasis. To prevent infection, all rats were treated with cefmetazole (Cefmetazon, Daiichi Sankyo, Tokyo, Japan, $40 \mathrm{mg} / \mathrm{kg} /$ day, subcutaneously) for 7 days. We evacuated the bladder of rats twice a day and used hind limb exercises to prevent contracture during the first 7 days after the surgery.

Two weeks after the spinal cord transection, rats were randomly divided into an SCS group and a control group. The rats in the SCS group underwent a laminectomy at the level of L2-3 and an SCS electrode (Pisces-Quad Lead, Medtronic, Minneapolis, MN) was implanted on the dorsal surface of the dural tube at the level of L2. The rats in the control group underwent a laminectomy at the level of L3-4 alone without implanting an electrode. 


\section{SCS and treadmill training}

Rats underwent SCS and treadmill training with body weight support for 30 min per day, 5 days per week, for four weeks. SCS was delivered at $40 \mathrm{~Hz} 240 \mu$ s pulses following a published protocol [4]. We adjusted intensities between 0.5 and $2.0 \mathrm{~V}$ under the threshold for generating hind limb locomotion.

Treadmill training was performed by using a device developed for the present study (Shibata system, Kyoto, Japan). The device had arms to passively move the hind limbs of the rats reciprocally. The device also had a harness for the rats that could be adjusted to support their body weight (Fig. 2). The training was performed with $95-100 \%$ of body weight support. Treadmill belt speed was set at $5 \mathrm{~cm} / \mathrm{s}$ and the arm of the device was adjusted to move the hind limbs of the rats in synchrony with the treadmill.

\section{Behavioral assessment}

We evaluated hind limb locomotor function using the Basso-Beattie-Bresnahan scale (BBB) score once a week for 6 weeks [9]. Rats were allowed to move freely in an open field for 5 min and their hind limb locomotion was assessed. We also measured resistance force for full extension of hind limbs to evaluate spasticity once a week for 6 weeks. In brief, we put the rats in a supine position in the harness and measured the maximum force by using a handheld strain gauge (model FGP-0.5; Nidec-Shimpo, Kyoto, Japan). Every measurement was repeated 6 times and the average was considered as the degree of spasticity.

\section{Immunohistochemistry}

To elucidate the possible mechanism for attenuation of spasticity obtained by SCS and treadmill training, we used immunohistochemistry to determine the expression of glutamic acid decarboxylase-65 (GAD65), a molecular marker for spinal cord inhibitory neurons.

Six weeks after spinal cord transection, the rats were humanely killed with an intraperitoneal overdose of pentobarbital sodium ( $50 \mathrm{mg} / \mathrm{kg}$ of Somnopentyl, Kyoritsuseiyaku, Tokyo, Japan) followed by transcardial perfusion with $4 \%$ paraformaldehyde. The lumbar enlargement of the spinal cord was removed and postfixed overnight in $4 \%$ paraformaldehyde. Tissues were stored in $20 \%$ sucrose overnight for cryoprotection and frozen in TissueTek OCT compound (Sakura Finetek, Tokyo, Japan) at $-70{ }^{\circ} \mathrm{C}$. Serial sections of the lumbar enlargement at $20 \mu \mathrm{m}$ thickness were cut on a cryostat and mounted onto MAS coated glass slides (Matsunami Glass Industries, Osaka, Japan).

The sections were permeabilized with $0.3 \%$ Triton X-100 (Nacalai Tesque, Kyoto, Japan) in PBS for $1 \mathrm{~h}$, then incubated with primary antibody (mouse anti-GAD65 antibody at $1: 200$, ab26113, Abcam) at $4{ }^{\circ} \mathrm{C}$ overnight. After washing with PBS three times, slides were incubated with secondary antibody (goat antimouse IgG $\mathrm{H}$ and $\mathrm{L}$ chain Alexa Fluor 488 at 1:200, ab150113, Abcam). GAD65 staining was visualized using fluorescence microscopy (BX51, Olympus, Tokyo, Japan), and assessed by observers blinded to the treatments who measured the numbers of GAD65-positive cells. 


\section{Statistical Analysis}

All data are presented as mean \pm I SEM. The differences in BBB score and spasticity between SCS and control groups were examined using a repeated measures ANOVA and relative GAD65 expression between SCS and control groups was examined using a $t$ test. All the analyses were conducted using JMP (version 10.0.2, SAS Institute, Cary, NC, USA); $p<0.05$ was considered statistically significant.

\section{Results}

\section{Behavioral assessment}

BBB score of all rats dropped to 0 points after spinal cord transection and did not change during the experimental period. There was no significant difference between the two groups (Fig. 3a).

There was no significant difference in the resistance force of the hind limb, which was assessed for spasticity, between the SCS and control groups two weeks after spinal cord transection $(0.13 \pm 0.01 \mathrm{~N}$ in the SCS group and $0.13 \pm 0.01 \mathrm{~N}$ in the control group). Six weeks after the spinal cord transection, the resistance of the hind limb in the SCS group was $0.28 \pm 0.01 \mathrm{~N}$, significantly lower than that in the control group (0.64 $\pm 0.03 \mathrm{~N}, p=0.048$ ) (Fig. 3b).

\section{Immunohistochemistry}

Semiquantitative analysis revealed that the level of expression of GAD65 was markedly greater in the SCS group than in the control group (Fig. 4, $p=0.05$ ).

\section{Discussion}

In the present study, SCS ameliorated spasticity of hind limb and induced upregulation of GAD65 in a rat model of SCl using spinal cord transection.

SCS with treadmill training improved spasticity. Previous reports showed that SCS is effective for spasticity related to damage to the brain or spinal cord and neurodegenerative disorders [11], multiple sclerosis [12], and spinal cord injury [13, 14]. Our findings were consistent with those previous reports.

The present study revealed that SCS in combination with treadmill training using body weight support induced upregulation of GAD65. GAD is an isozyme involved in the synthesis of $y$-aminobutyric acid (GABA) and plays a central role in modulation of GABA synthesis. GAD65 is mainly found in membranes and nerve endings and may synthesize GABA for vesicular release [15]. GAD65 is known to decrease in rat models of $\mathrm{SCl}$ using spinal cord transection and may be related to the rhythmic inhibitory input to motoneurons during locomotion $[16,17]$. Therefore, upregulation of GAD65 is one of the possible mechanisms underlying the SCS-induced attenuation of spasticity. 
In the present study, we failed to improve the hind limb locomotor activity by SCS in combination with treadmill training using body weight support after spinal cord transection in adult rats. There are several possible explanations for this failure. First, spinal cord complete transection might be too severe to observe slight recovery of spinal cord function. Previous reports describing the efficacy of SCS on locomotion in rats and human used complete SCI models or patients with incomplete SCl. Ichiyama et al. reported that epidural SCS induced bilateral stepping patterns [4] and that combined therapy with the 5$\mathrm{HT}$ agonist quipazine and step training was effective in a rat model of complete $\mathrm{SCl}[5,6]$. Herman et al. reported that combined epidural SCS and partial weight bearing therapy could facilitate restoration of functional locomotion in patients with an AIS C classification, in whom motor palsy was incomplete at the initiation of the experimental treatment. This patient showed improvement of short distance walking without SCS after a few months of training [7]. Harkema et al. reported that the epidural SCS for CPG enabled full weight-bearing standing and stepping in a patient with an AIS B rating, whose palsy was incomplete and who had remaining sensory function even though motor palsy was complete [8]. The reason why we used a spinal cord transection model was to exclude the influence from supraspinal pathways and to assess solely the effect of SCS for CPG. It is well known that a model using spinal cord transection departs from $\mathrm{SCl}$ in humans because $\mathrm{SCl}$ is commonly caused by blunt trauma and spinal cord transection is an extremely rare situation in the clinic. Application of spinal cord contusion/compression models, which are the criterion standard for SCl research, may address this issue.

The other possible factors contributing to the failure of functional recovery in the present study are the location and mode of the SCS. We applied the stimulation in accordance with the previous reports including that by Ichiyama et al., who reported that the dorsal region of the L2 spinal segment was a particularly effective stimulation site to induce functional recovery of the hind limb. We applied the stimulation parameters set by these investigators ( $40 \mathrm{~Hz}$ and $0.5-2.0 \mathrm{~V}$ ). However, the optimal stimulation parameters warrant further exploration.

\section{Conclusions}

A combination of SCS and treadmill training with body weight support in a model of SCl using spinal cord transection can contribute to improve the hind limb function after $\mathrm{SCl}$, although further exploration is needed to prove the efficacy of this treatment for the other models of $\mathrm{SCl}$ and to elucidate the underlying mechanism in more detail.

The combination of SCS and treadmill training with body weight support ameliorated hind limb spasticity in rats with a spinal cord transection. Upregulation of GAD65 may be involved in suppression of spasticity. We failed to improve the hind limb locomotor activity by SCS in combination with treadmill training using body weight support.

\section{Abbreviations}


AIS: American Spinal Injury Association Impairment Scale, BBB: Basso-Beattie-Bresnahan, CPG: central pattern generator, GABA: g-aminobutyric acid, GAD65: glutamic acid decarboxylase-65, OCT: Optimal cutting temperature, PBS: phosphate buffered saline, SCI: spinal cord injury, SCS: spinal cord stimulation

\section{Declarations}

\section{Ethic approval and consent to participate}

This study was approved by the ethical committee of Chiba University Hospital. (approval No. A28-090, 29-10)

\section{Consent for publication}

Not applicable.

\section{Availability of data and materials}

The datasets used during the current study are available from the corresponding author on reasonable request.

\section{Competing interests}

The authors declare that they have no competing of interest in relation to this research and its publication.

\section{Funding}

This study was supported by grants-in-aid for Scientific Research (C) from the Ministry of Education, Culture, Sports, Science and Technology of Japan (17K10922).

\section{Author's Contributions}

JS, MaK, TF, MY and SO conceived of the study. JS, YI, MiK, TM and CK performed the experiments. JS, $\mathrm{YI}, \mathrm{MiK}$ and TM analyzed the data. JS drafted the manuscript. MaK, TF, SO, KI, MY and SO revised the manuscript critically and approved the modified text. All authors have read and approved the final manuscript. 


\section{Acknowledgements}

This study was supported by grants-in-aid for Scientific Research (C) from the Ministry of Education, Culture, Sports, Science and Technology of Japan (17K10922).

\section{References}

1. Dimitrijevic MR, Gerasimenko Y, Pinter MM. Evidence for a spinal central pattern generator in humans. Ann NY Acad Sci. 1998;16:360-76.

2. Brown TG. The intrinsic factors in the act of progression in the mammal. Proc. R. Soc. Lond. 1911;84B:308-319.

3. Majczynski H, Slawinska U. Locomotor recovery after thoracic spinal cord lesions in cats, rats and humans. Acta Neurobiol Exp. 2007;67:235-57.

4. Ichiyama RM, Gerasimenko YP, Zhong H. Hindlimb stepping movements in complete spinal rats induced by epidural spinal cord stimulation. Neurosci Lett. 2005;383:339-44.

5. Ichiyama RM, Courtine G, Gerasimenko YP. Step training reinforces specific spinal locomotor circuitry in adult spinal rats. J Neurosci. 2008;28:7370-5.

6. Ichiyama RM, Gerasimenko Y, Jindrich DL. Dose dependence of the 5-HT agonist quipazine in facilitating spinal stepping in the rat with epidural stimulation. Neuroscience Lett. 2008;438:281-5.

7. Herman R, He J, D'Luzansky S. Spinal cord stimulation facilitates functional walking in a chronic, incomplete spinal cord injured. Spinal Cord. 2002;40:65-8.

8. Harkema S, Gerasimenko Y, Hodes J. Effect of epidural stimulation of the lumbosacral spinal cord on voluntary movement, standing, and assisted stepping after motor complete paraplegia: a case study. Lancet. 2011;377:1938-47.

9. Basso DM, Beattie MS, Bresnahan JC. A sensitive and reliable locomotor rating scale for open field testing in rats. J Neurotrauma. 1995;12:1-21.

10. Baker D, Pryce G, Croxford JL. Cannabinoids control spasticity and tremor in a multiple sclerosis model. Nature. 2000;404:84-7.

11. Nagel SJ, Wilson S, Johnson MD. Spinal cord stimulation for spasticity: historical approaches, current status, and future directions. Neuromodulation. 2017;20:307-21.

12. Cook AW, Weinstein SP. Chronic dorsal column stimulation in multiple-sclerosis: preliminary report. NY State J Med. 1973;73:2868-72.

13. Dimitrijevic MM, Dimitrijevic MR, Illis LS. Spinal cord stimulation for the control of spasticity in patients with chronic spinal cord injury: I. Clinical observations. Cent Nerv Syst Trauma. 1986;3:12943.

14. Barolat G, Singh-Sahni K, Staas WE Jr, Shatin D, Ketcik B, Allen K. Epidural spinal cord stimulation in the management of spasms in spinal cord injury: a prospective study. Stereotact Funct Neurosurg. 
1995;64:153-64.

15. Soghomonian JJ, Martin DL. Two isoforms of glutamate decarboxylase. why? Trends Pharmacol Sci. 1998;19:500-5.

16. Corleto JA, Bravo-Hernandez M, Kamizato K. Thoracic 9 spinal transection-induced model of muscle spasticity in the rat: a systematic electrophysiological and histopathological characterization. PLoS One. 2015;10.

17. Wilson JM, Blagovechtchenski E, Brownstone RM. Genetically defined inhibitory neurons in the mouse spinal cord dorsal horn: a possible source of rhythmic inhibition of motoneurons during fictive locomotion. J Neurosci. 2010;30:1137-48.

\section{Figures}

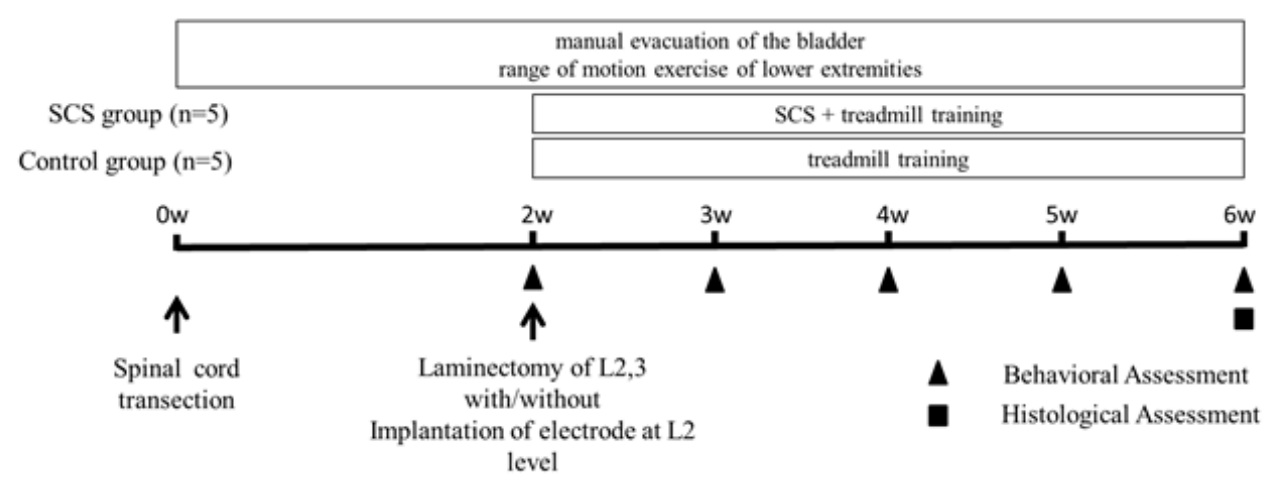

Figure 1 
Experimental protocol. We transected the spinal cord at the level of T8-9. After two weeks, the rats in the SCS group were implanted with an electrode at the level of L2. SCS and treadmill training with body weight support were performed for 30 min per day, 5 days per week, for 4 weeks. The rats in the control group underwent the treadmill training without SCS for the same period. We assessed hind limb locomotor function using the BBB score and resistance force measurements for full extension of the hind limb to evaluate spasticity every week. After four weeks of treadmill training with or without SCS, we immunostained the lumbar enlargement for GAD65.

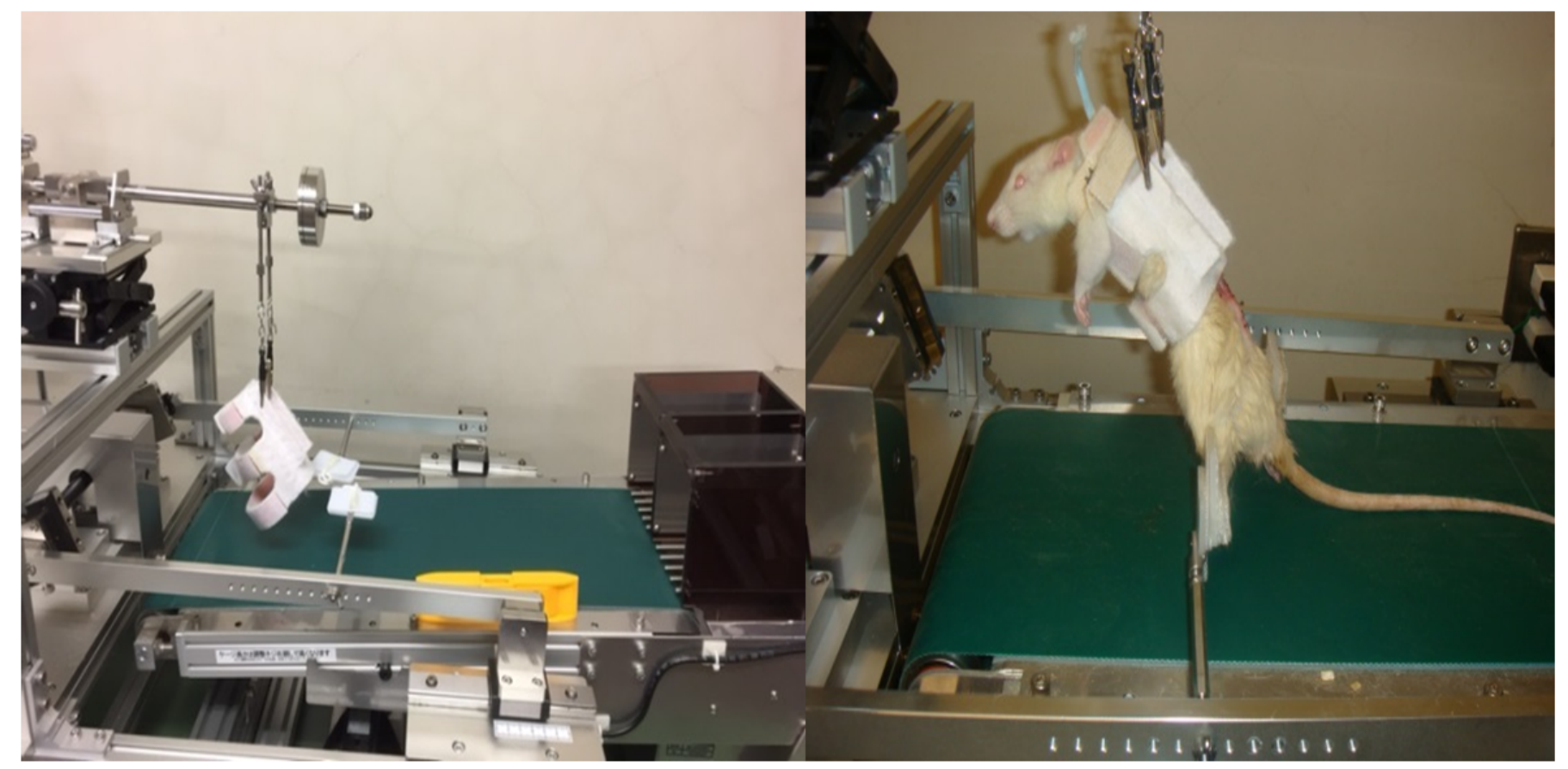

\section{Figure 2}

The device for treadmill training. The device has arms and moves the hind limbs of the rats one after the other. The device also has a harness for rats and could control their body weight support. The present training was performed with $95 \%$ to $100 \%$ of body weight support. Treadmill belt speed was set $5 \mathrm{~cm} / \mathrm{s}$ adjusted for device motion. 


\section{BBB score}

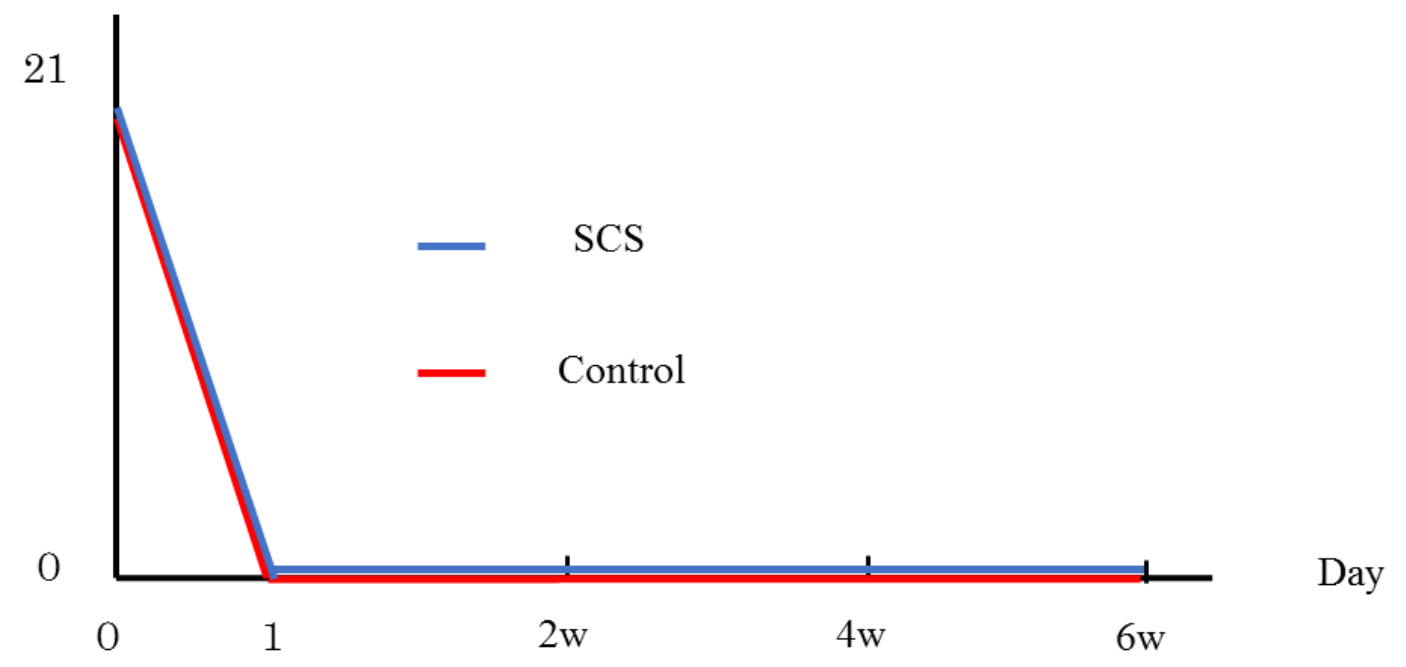

Figure 3

BBB scores. After spinal cord transection, BBB score of all rats was 0 . BBB score did not change up to six weeks. 


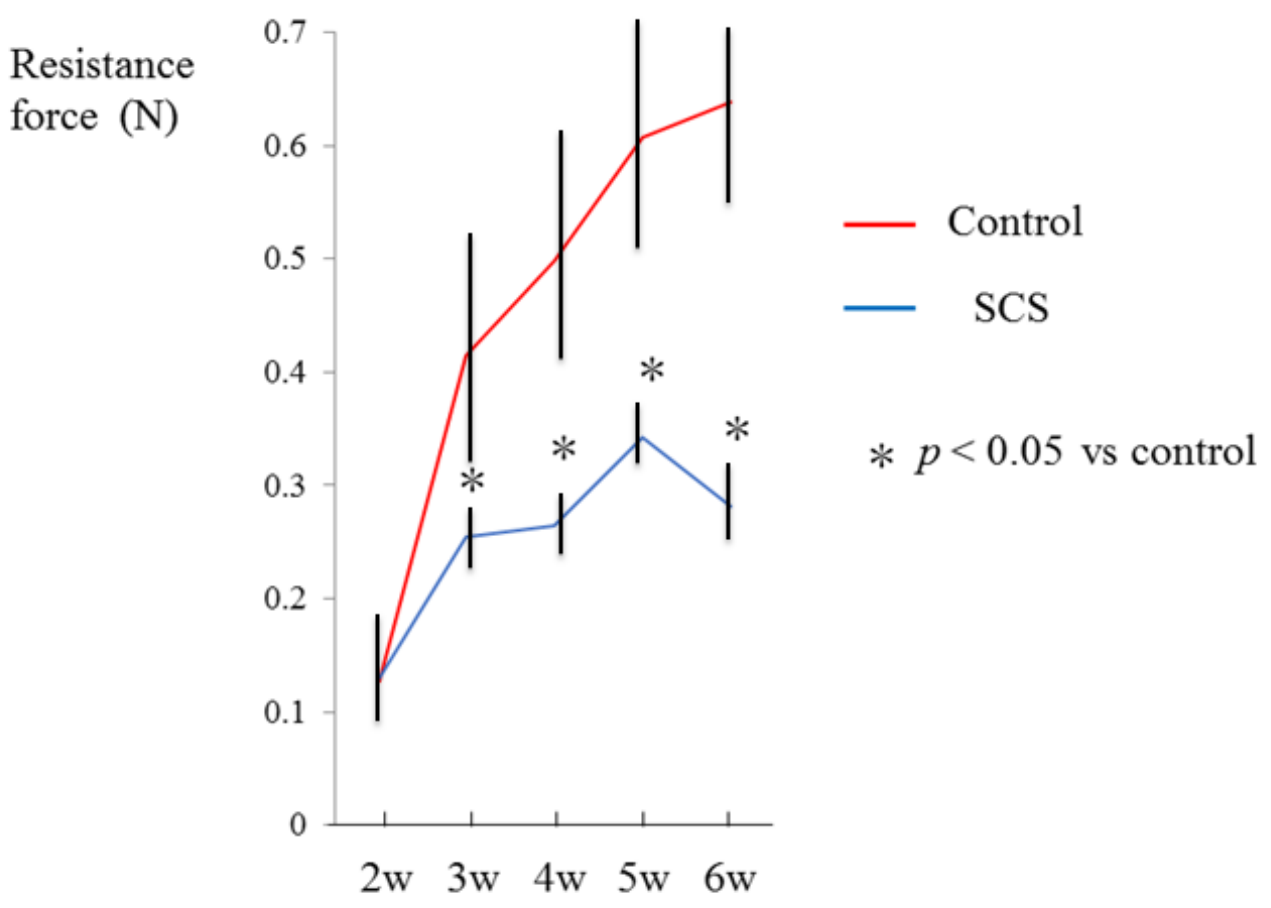

Figure 4

Resistance force for full extension. There was no significant difference between the two groups at two weeks after spinal cord transection. At six weeks after spinal cord transection, the resistance force of the hind limb in SCS and control groups were $0.28 \pm 0.06 \mathrm{~N}$ and $0.64 \pm 0.16 \mathrm{~N}$. There was a significant reduction of spasticity in the SCS group $(p=0.048)$. 

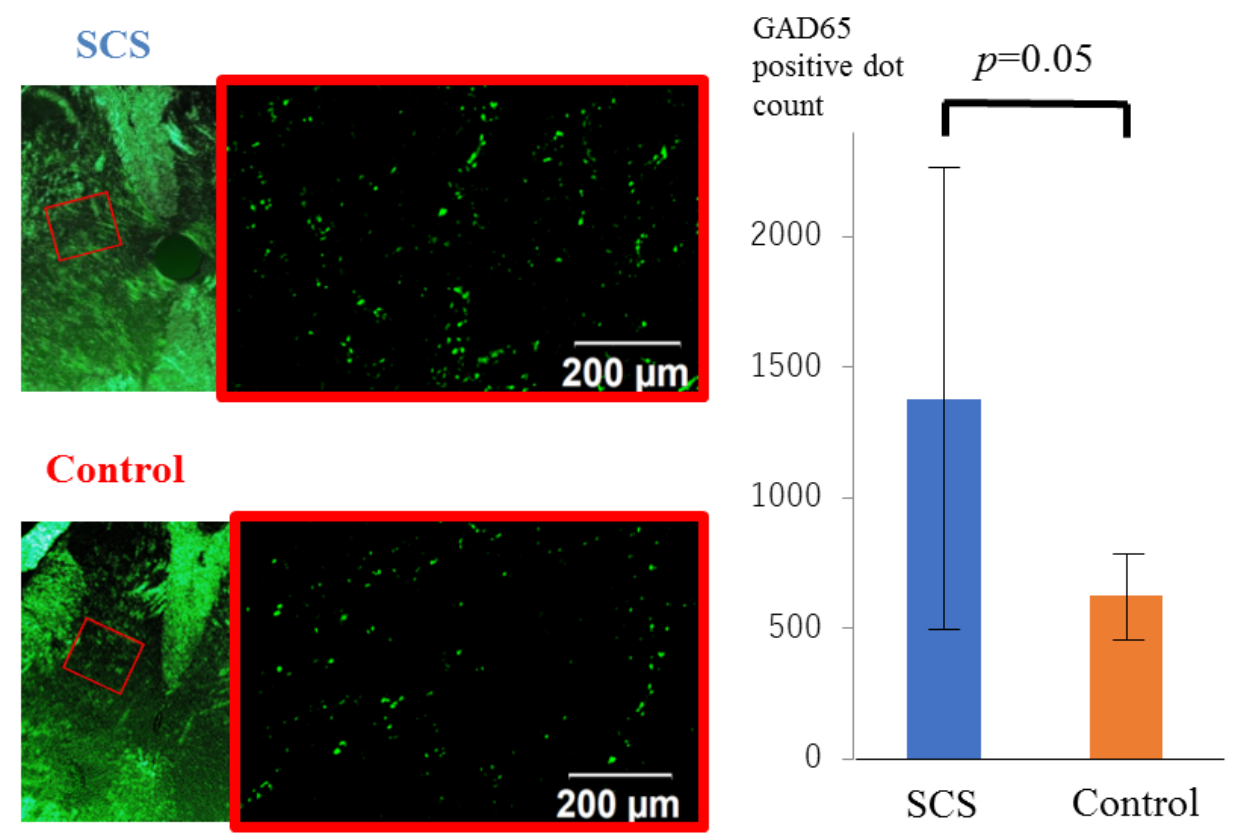

\section{Figure 5}

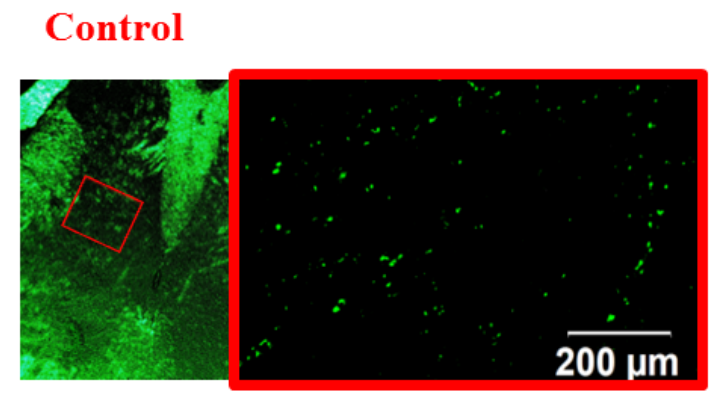

Histology. Quantitative analysis of GAD65 by an investigator blinded to treatments showed no significant difference between the two groups $(p=0.05)$, but expression of GAD65 tended to be greater in the SCS group than controls.

\section{Supplementary Files}

This is a list of supplementary files associated with this preprint. Click to download.

- NC3RsARRIVEGuidelinesChecklistfillable.pdf 